\title{
Monoclonal antibody against CXCL-10/IP-10 ameliorates influenza A (H1N1) virus induced acute lung injury
}

Cell Research (2013) 23:577-580. doi:10.1038/cr.2013.25; published online 19 February 2013

\section{Dear Editor,}

Most patients infected with 2009 swine-origin influenza A (H1N1) virus (S-OIV) suffered respiratory illness. Some severely infected patients developed acute lung injury (ALI) and even acute respiratory distress syndrome (ARDS), which is the predominant cause for reported deaths [1]. After the pandemic, an analysis of data from flu cases in adults and children living in Wisconsin state of USA concluded that the symptoms and risk of complications from 2009 H1N1 swine flu was not greater compared with either H1N1 or H3N2 seasonal flu [2]. The H1N1 pandemic flu was not linked to substantially more hospitalization or pneumonia than these recent seasonal flu strains [2]. However, a recent study estimated that the mortality associated with the $2009 \mathrm{H} 1 \mathrm{~N} 1$ swine flu was 15 times higher than the World Health Organization (WHO) reported [3], confirming that the 2009 S-OIV outbreak was a pandemic.

Previous studies showed that excessive early cytokine responses with early recruitment of inflammatory immune cells to the lung were key contributors to the morbidity of the 1918 influenza and avian H5N1 infection [4-6]. In patients with 2009 S-OIV H1N1 infection, plasma levels of proinflammatory cytokines and chemokines were higher among those who experienced fatal ARDS compared with those with non-fatal ARDS or mild SOIV H1N1 illness [7]. Systemic levels of immune mediators associated with $\mathrm{T}$ helper 1 and $\mathrm{T}$ helper 17 responses were elevated in hospitalized patients infected with SOIV H1N1, but not in outpatients with mild S-OIV H1N1 illness [8]. C-X-C motif chemokine 10 (CXCL10), also known as interferon $\gamma(\mathrm{IFN}-\gamma)$-induced protein 10 $\mathrm{kDa}$ (IP-10), was found to be significantly elevated in the serum of patients with ARDS induced by S-OIV H1N1 infection $[7,8]$. IP-10 is a proinflammatory cytokine belonging to the CXC chemokine family. After binding its unique receptor CXCR3, the seven transmembranespanning G protein-coupled receptor, IP-10 induces chemotaxis in different cell types of the immune system and plays an important role in the innate immune response.
This is crucial for the initiation and subsequent direction of adaptive immune responses, as well as in promoting localized inflammation and producing cytokines that recruit additional leukocytes to the site of infection. In addition, previous studies showed that the proliferation of $\mathrm{T}$ cells upon allogeneic and antigenic stimulation and the secretion of IFN- $\gamma$ in response to antigenic challenge were impaired in IP-10-deficient mice, suggesting that IP-10 plays an important role in T-cell attraction and activation [9]. Alterations in the IP-10 expression level have been shown to be associated with inflammatory diseases and tissue damage [10]. However, the functional role and molecular mechanism of IP-10 in the ALI induced by influenza virus infection remain unclear.

During the 2009 swine flu pandemic, we recruited dozens of patients who were PCR positive for the 2009 S-OIV H1N1 and 9 patients with normal upper respiratory tract infections from two hospitals in Beijing, China [11]. We performed a Bio-plex cytokine assay on the serum samples from 23 qualified patients. The concentration of IP-10 in the serum of the 9 critical patients with ARDS was significantly elevated compared with the control group (Figure 1A), confirming the previous reports [7, 8].

We created an ALI model in mice infected with the A/Beijing/501/2009 (H1N1) (BJ501) strain isolated in China during the 2009 pandemic [11]. We measured the concentration of IP-10 in the bronchoalveolar lavage fluid (BALF) of BJ501 strain-infected wild-type (WT) mice and found that the IP-10 level was also significantly increased (Supplementary information, Figure S1A), confirming the important role of IP-10 in mediating ALI induced by 2009 S-OIV H1N1 infection.

To elucidate the functional role of IP-10 in ALI, we infected the IP-10-deficient mice with the BJ501 strain and found that the survival rate of the mice was significantly improved compared with the infected WT mice (Figure 1B), and body weight losses in IP-10-deficient mice were reduced (Figure 1C). The pulmonary edema measured by wet-to-dry lung weight ratio in IP-10-deficient mice was significantly ameliorated (Figure 1D). The lung histopa- 
A

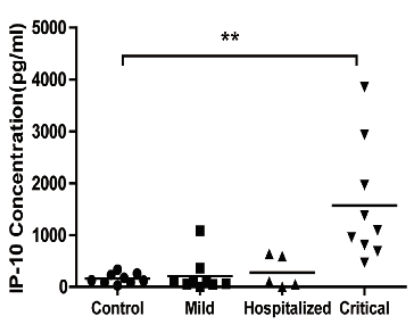

B
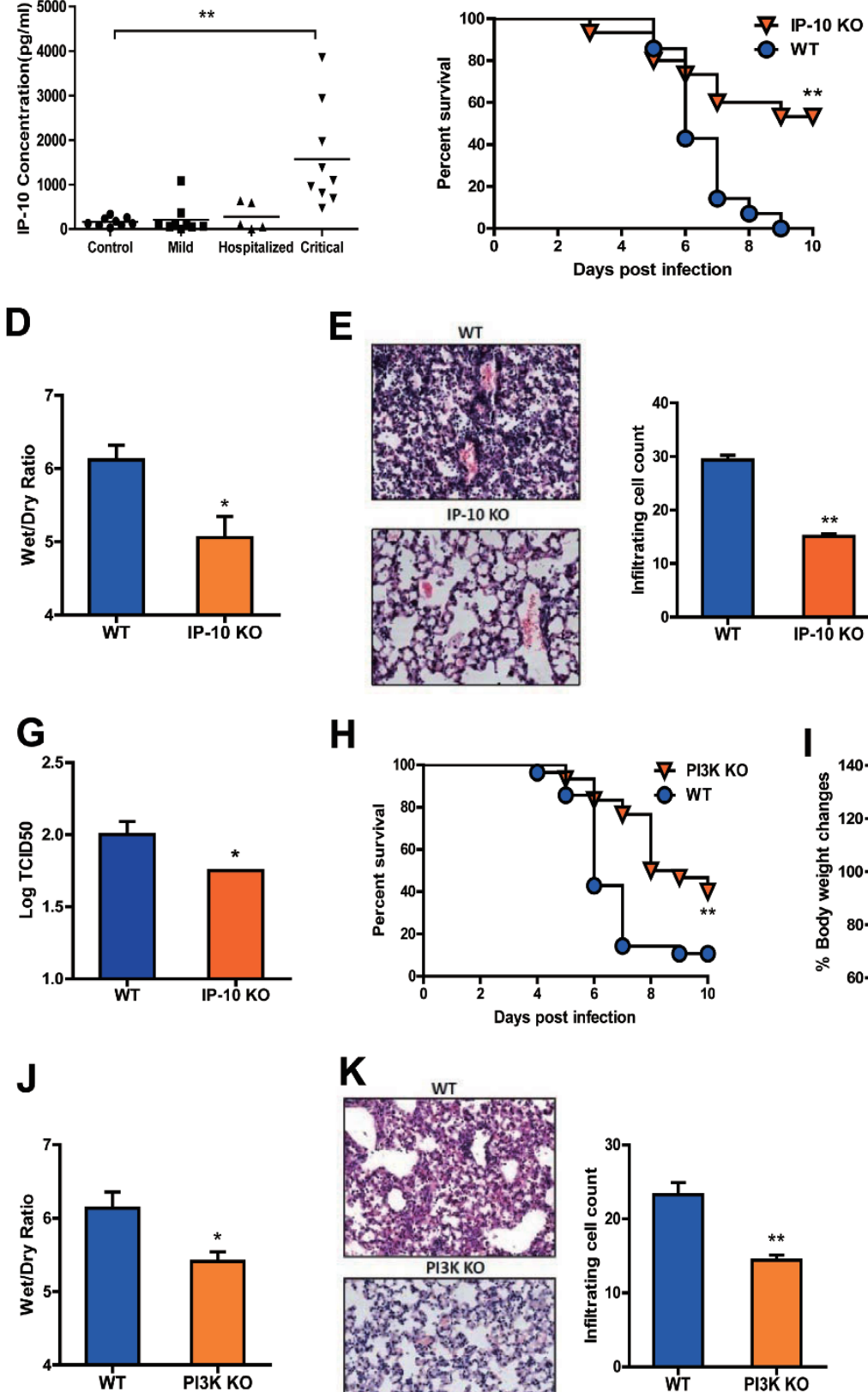

M

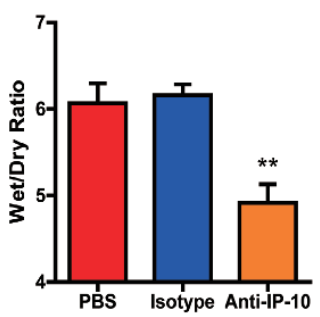

E

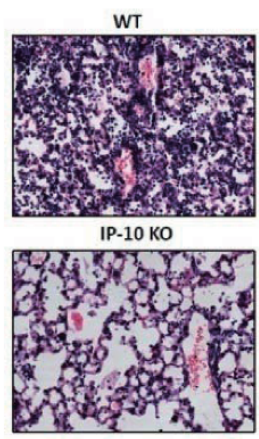

H
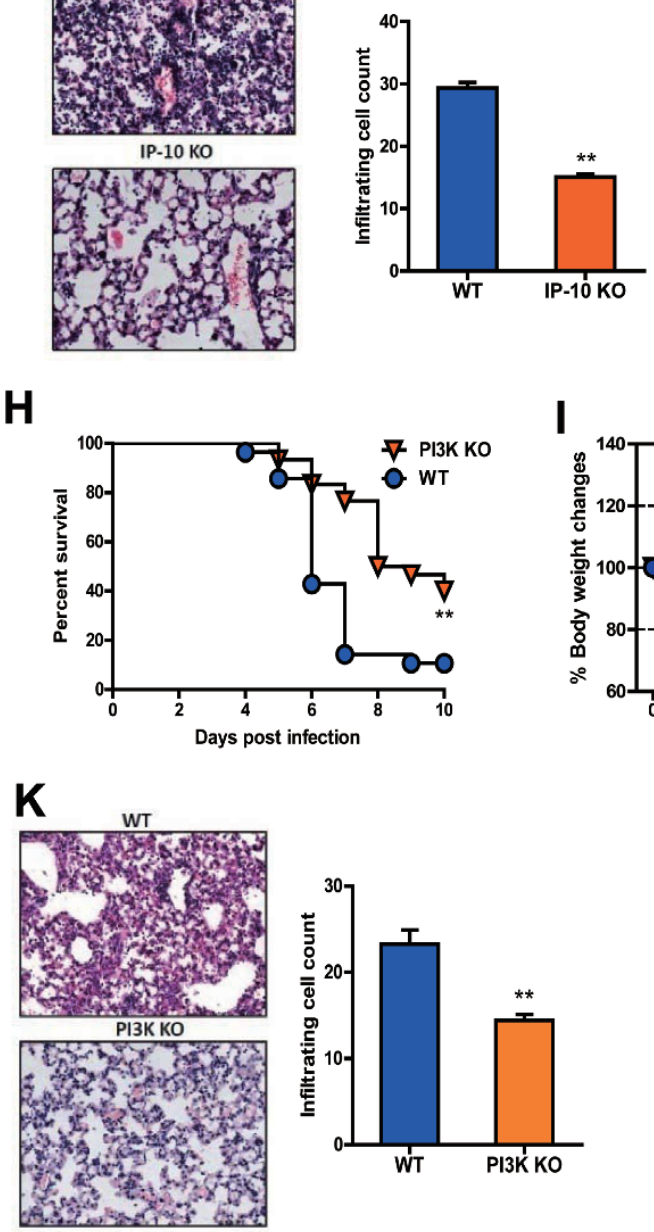

I

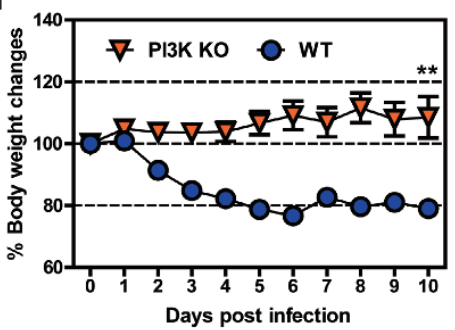

L

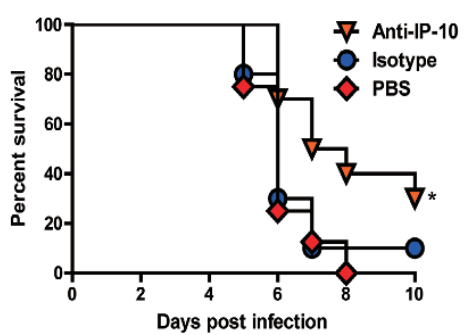

N
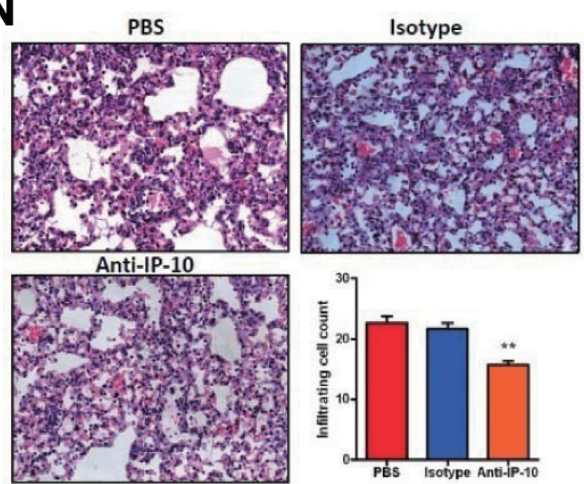

O

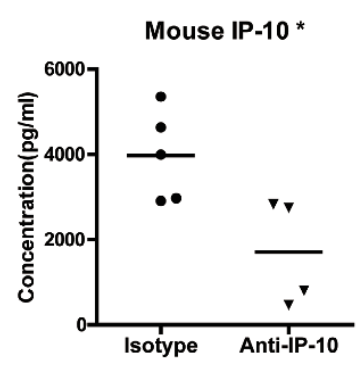

F

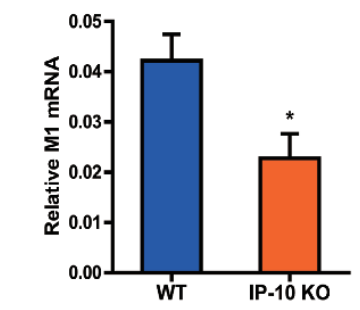

C

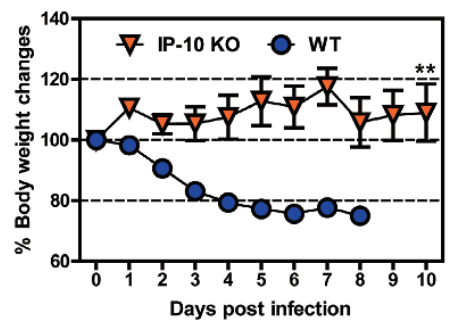


Figure 1 IP-10 plays a critical role in the ALI induced by 2009 S-OIV H1N1. (A) The concentration of IP-10 in the sera from patients infected with 2009 S-OIV H1N1 was determined using a Bio-Plex Human Cytokine Array. 4-week-old mice were anesthetized with $50 \mu \mathrm{l} 1 \%(\mathrm{w} / \mathrm{v})$ pentobarbital sodium and intranasally treated with $10^{5.5}$ TCID50 of BJ501 H1N1 virus. (B)

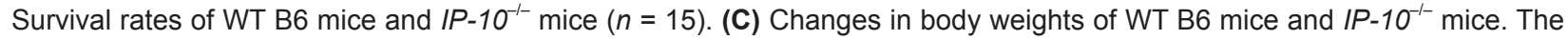
values are means \pm SEM from 15 mice. (D) Wet-to-dry ratios of lung of WT B6 mice and $I P-10^{-1-}$ mice $(n=6)$ at 5 days post infection (DPI). (E) HE staining and infiltrating cell count $\left(n=50\right.$ fields) in lung tissue of WT B6 mice and $I P-10^{-1-}$ mice $(n=3)$

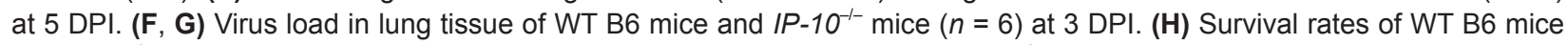
and $P I 3 K^{-1-}$ mice $(n=30)$. (I) Changes in body weights of WT B6 mice and $P / 3 K^{-1-}$ mice. The values are means \pm SEM from 30 mice. (J) Wet-to-dry ratios of lungs from WT B6 mice and $P / 3 K^{-1-}$ mice $(n=8)$ at $5 \mathrm{DPI}$. (K) HE staining and infiltrating cell count $\left(n=50\right.$ fields) in lung tissue of WT B6 mice and PI3K $K^{--}$mice $(n=3)$ at 5 DPI. 4-week-old WT B6 mice were treated intravenously with $50 \mu \mathrm{g}$ of anti-IP-10 or isotype-control antibodies per mouse 1 day before infection of BJ501 strain and at 1 DPI and 3 DPI. (L) Survival rates of treated B6 mice $(n=10)$. (M) Wet-to-dry ratios of lungs from treated B6 mice $(n=5)$ at 5 DPI. (N) HE staining and infiltrating cell count $(n=50$ fields) in lung tissue of treated B6 mice $(n=3)$ at $5 \mathrm{DPI}$. (O) Concentration of IP-10 in the BALFs of treated B6 mice $(n=5)$ at 24 h post-infection. Magnification, $200 \times .{ }^{*} P<0.05$ and ${ }^{* \star} P<0.01$. All experiments were repeated at least three times.

thology of IP-10-deficient mice was greatly improved, and the leukocyte infiltration cell counts significantly decreased (Figure 1E). In addition, the virus load of infected IP-10-deficient mice was significantly decreased compared with that of WT mice (Figure $1 \mathrm{~F}$ and $1 \mathrm{G})$. To test whether this finding is common in other influenza virus infections, we infected the IP-10-deficient mice with the mouse-adapted influenza A/Puerto Rico/8/34 (PR8) strain and obtained similar results (Supplementary information, Figure S2). Taken together, these results suggest that IP-10 elevation is critical in influenza virus infection-induced mouse ALI.

To further elucidate the signaling pathways through which IP-10 may lead to ALI, we infected IP-10-deficient mice with the 2009 pandemic influenza BJ501 strain and performed western blot analysis. We found that the Akt-p38-ATF2 pathway was involved in BJ501 strain-induced ALI (Supplementary information, Figure S4A). PI3K is upstream of the Akt-p38-ATF2 pathway. We then infected the PI3K-deficient mice with BJ501 strain and found that the ALI was significantly ameliorated compared with the infected WT mice (Figure 1H, $1 \mathrm{I}, 1 \mathrm{~J}, 1 \mathrm{~K})$. We obtained similar results in the PR8 straininfected PI3K-deficient mice (Supplementary information, Figure S3). These data indicate that the PI3K-Aktp38-ATF2 signaling pathway may be involved in the ALI mediated by IP-10 in BJ501 strain-infected mice. We also found that the JNK/MAPK signaling pathway was significantly downregulated in the BJ501 strain-infected IP-10-deficient mice compared with the control mice (Supplementary information, Figure S4B), suggesting that the JNK/MAPK signaling pathway may also play a critical role in IP-10-mediated ALI by BJ501 infection in mice. A proposed schematic model of IP-10 signaling in the regulation of ALI in our BJ501 strain-infected mouse model is shown (Supplementary information, Figure
S4C), providing potential targets for the treatment of ALI induced by S-OIV H1N1 infection.

Although corticosteroid was shown effective in treating mouse ALI induced by 2009 S-OIV H1N1 infection [12], modulation of the host immune response with less selective pressure on viral populations is enticing. MDX1100 , a fully human anti-IP-10 monoclonal antibody, is well tolerated and demonstrated clinical efficacy in patients with rheumatoid arthritis who responded inadequately to Methotrexate in a phase II, randomized, double-blind, placebo-controlled study [13]. To test whether monoclonal antibody against IP-10 could protect against the ALI induced by the 2009 S-OIV H1N1 infection, we treated BJ501 strain-infected mice with monoclonal antibody against human IP-10. We found that the survival rate of the infected mice treated with antiIP-10 monoclonal antibody was significantly increased compared with isotype control-treated mice (Figure 1L). The formation of lung edema and lung histopathology alterations in the anti-IP-10 monoclonal antibody-treated mice were significantly improved (Figure $1 \mathrm{M}$ and $1 \mathrm{~N}$ ). After treatment with anti-IP-10 monoclonal antibody, the levels of responsive cytokines and chemokines were also significantly reduced in the infected mice compared with the infected mice treated with isotype control (Figure 10 and Supplementary information, Figure S5A). We then treated PR8 strain-infected mice with the monoclonal antibody against human IP-10 and found that it could also significantly ameliorate the ALI induced by PR8 strain infection (Supplementary information, Figure S6). Altogether, these data suggest that therapeutic anti-IP-10 monoclonal antibody treatment may be a useful remedy for the future influenza A (H1N1) virus pandemic.

A recent study demonstrated that IP-10 response enhanced the development of neutrophil-mediated fulminant lung injury of viral and non-viral origin [14]. Simi- 
lar to our results, they found that IP-10 was elevated in sera of patients with ARDS and the mice lacking IP-10 or CXCR3 demonstrated improved severity and survival of non-viral and viral ALI. They further identified that neutrophil was the main source of IP-10 production in the fulminant pulmonary inflammation. However, our data provide the molecular signaling mechanism linking ALI pathogenesis and the role of IP-10 in lung failure. To our knowledge, we are the first to systematically demonstrate that PI3K-Akt-p38-ATF2 and JNK/MAPK signaling pathways are involved in the ALI mediated by IP-10 in 2009 S-OIV H1N1-infected mice. As the elevation of IP10 level was also found in the serum of patients infected with SARS, avian H5N1 influenza virus, and hepatitis $\mathrm{C}$ virus $[5,10,15]$, our data may assist in elucidating the functional role of IP-10 in the tissue pathogenesis of these viral infections. Moreover, our results show that treatment with monoclonal antibodies against IP-10 improved the survival rate and ameliorated the ALI induced by influenza A (H1N1) virus infection in mice, revealing an effective approach to achieve immunotherapy for any future influenza A (H1N1) virus pandemic. Detailed methods are described in the Supplementary information, Data S1.

\section{Acknowledgments}

This work is supported by the Ministry of Science and Technology of China (2009CB522105), the National Natural Science Foundation of China (81030032, 81230002), and the 111 Project (B08007). CJ is a Hsien Wu professor of Biochemistry.

Wei Wang ${ }^{1, *}$, Penghui Yang, ${ }^{2,3,}$, Ying Zhong ${ }^{1, *}$, Zhongpeng Zhao ${ }^{2}$, Li Xing ${ }^{2}$, Yan Zhao ${ }^{1}$, Zhen Zou ${ }^{1}$, Yanli Zhang ${ }^{1}$, Chenggang $\mathrm{Li}^{1}$, Taisheng $\mathrm{Li}^{4}$, Chen Wang ${ }^{5,6}$, Zhong Wang ${ }^{4}$, Xuezhong Yu, Bin $\mathrm{Cao}^{6}$, Xiang $\mathrm{Gao}^{7}$, Josef M Penninger ${ }^{8}$, Xiliang Wang ${ }^{2}$, Chengyu Jiang ${ }^{1,4}$
${ }^{I}$ State Key Laboratory of Medical Molecular Biology, Institute of Basic Medical Sciences, Chinese Academy of Medical Sciences, Department of Biochemistry and Molecular Biology, Peking Union Medical College, Tsinghua University, Beijing 100005, China; ${ }^{2}$ State Key Laboratory of Pathogens and Biosecurity, Beijing Institute of Microbiology and Epidemiology, Beijing 100071, China; ${ }^{3}$ Department of Hepatobiliary, 302 Military Hospital, Beijing 100039, China; ${ }^{4}$ Center for Translational Medicine, Peking Union Medical College Hospital, Beijing 100730, China; ${ }^{5}$ Beijing Hospital, China Ministry of Health, Beijing 100730, China $;{ }^{6}$ Beijing ChaoYang Hospital, Beijing Institute of Respiratory Medicine, Capital Medical University, Beijing 100020, China; ${ }^{7}$ MOE key laboratory of Model Animal for Disease Study, Model Animal Research Center of Nanjing University, Nanjing, Jiangsu 210061, China; ${ }^{8}$ Institute of Molecular Biotechnology in the Austrian Academy of Sciences, Vienna A-1030, Austria

*These three authors contributed equally to this work.

Correspondence: Chengyu Jiang ${ }^{\mathrm{a}}$, Xiliang Wang ${ }^{\mathrm{b}}$

${ }^{\mathrm{a} T e l}$ : +86-10-65296908; Fax: +86-10-65276551

E-mail: jiang@pumc.edu.cn

bE-mail: xiliangw@126.com

\section{References}

1 Louie JK, Acosta M, Winter K, et al. JAMA 2009; 302:1896-1902.

2 Belongia EA, Irving SA, Waring SC, et al. JAMA 2010; 304:10911098.

3 Dawood FS, Iuliano AD, Reed C, et al. Lancet Infect Dis 2012; 12:687-695.

4 Kobasa D, Jones SM, Shinya K, et al. Nature 2007; 445:319-323.

5 de Jong MD, Simmons CP, Thanh TT, et al. Nat Med 2006; 12:12031207.

6 Wang H, Jiang C. Sci China C Life Sci 2009; 52:464-469.

7 To KK, Hung IF, Li IW, et al. Clin Infect Dis 2010; 50:850-859.

8 Bermejo-Martin JF, Ortiz de Lejarazu R, Pumarola T, et al. Crit Care 2009; 13:R201.

9 Dufour JH, Dziejman M, Liu MT, et al. J Immunol 2002; 168:31953204.

10 Reiberger T, Aberle JH, Kundi M, et al. Antivir Ther 2008; 13:969976.

11 Li C, Yang P, Sun Y, et al. Cell Res 2012; 22:528-538.

12 Li C, Yang P, Zhang Y, et al. PloS One 2012; 7:e44110.

13 Yellin M, Paliienko I, Balanescu A, et al. Arthritis Rheum 2012; 64:1730-1739.

14 Ichikawa A, Kuba K, Morita M, et al. Am J Respir Crit Care Med 2013; 187:65-77.

15 Tang NL, Chan PK, Wong CK, et al. Clin Chem 2005; 51:2333-2340.

(Supplementary information is linked to the online version of the paper on the Cell Research website.) 